\title{
MULTIWAVELENGTH OPTICAL OBSERVATIONS OF TWO CHROMOSPHERICALLY ACTIVE BINARY SYSTEMS: V789 MON AND GZ LEO*
}

\author{
M. C. Gálvez ${ }^{1,2}$, D. Montes ${ }^{2}$, M. J. Fernández-Figueroa ${ }^{2}$, E. De Castro ${ }^{2}$, and M. Cornide ${ }^{2}$ \\ ${ }^{1}$ Centre for Astrophysics Research, Science and Technology Research Institute, University of Hertfordshire, Hatfield AL10 9AB, UK \\ ${ }^{2}$ Departamento de Astrofísica, Facultad de Ciencias Físicas, Universidad Complutense de Madrid, E-28040 Madrid, Spain; mcz@astrax.fis.ucm.es \\ Received 2007 December 11; accepted 2009 January 7; published 2009 March 10
}

\begin{abstract}
This paper describes a multiwavelength optical study of chromospheres in two X-ray/EUV-selected active binary stars with strong $\mathrm{H} \alpha$ emission, V789 Mon (2RE J0725-002) and GZ Leo (2RE J1101+223). The goal of the study is to determine radial velocities and fundamental stellar parameters in chromospherically active binary systems in order to include them in the activity-rotation and activity-age relations. We carried out high-resolution echelle spectroscopic observations and applied spectral-subtraction technique in order to measure emission excesses due to chromosphere. The detailed study of activity indicators allowed us to characterize the presence of different chromospheric features in these systems and enabled to include them in a larger activity-rotation survey. We computed radial velocities of the systems using cross-correlation with the radial velocity standards. The double-line spectral binarity was confirmed and the orbital solutions improved for both systems. In addition, other stellar parameters such as spectral types, projected rotational velocities $(v \sin i)$ and the equivalent width of the lithium Li I $\lambda 6707.8$ $\AA$ Absorption line were determined.
\end{abstract}

Key words: binaries: spectroscopic - stars: activity - stars: individual (V789 Mon, GZ Leo)

Online-only material: color figure

\section{INTRODUCTION}

Why each particular star reaches a certain level of activity, and why seemingly similar stars may have drastically different chromospheres? It is believed that differential rotation and convection are the key factors responsible for the magnetic activity in the star. A significant fraction of stellar population, especially at later evolutionary stages, is found in close binaries, where tidal forces and mass transfer become additional factors controlling rotation and atmospheric properties of companions.

In order to understand the physical origin of activity, the timescale of variability, and to be able to extrapolate to other systems, we initiated a multiwavelength optical survey of a large sample of binaries with various levels of activity. We carried out the study of the chromosphere of active binary systems, using the information provided by several optical and near-infrared spectroscopic features that are formed at different heights in the chromosphere (see Montes et al. 1997, 1998, 2000; Gálvez et al. 2002, 2007), and applied it to several known and newly discovered binaries, most of which are BY Dra or RS CVn type with various levels of activity.

The ultimate goal of the project is to establish a reliable activity-rotation relation in binary stars. To achieve high accuracy, one needs to investigate all possible causes of activity for each particular system and for each component in the system. It is essential to take into consideration dispersion and signal to noise of the spectral data obtained with different instrumental setups. Furthermore, the study of binaries as compared to single stars is complicated by the interaction between companions, line blending (SB2 case), reflected light, etc. Active stars, which

\footnotetext{
* Based on observations collected with the $2.2 \mathrm{~m}$ telescope at the Centro Astronómico Hispano Alemán (CAHA) at Calar Alto (Almería, Spain), operated jointly by the Max-Planck Institut für Astronomie and the Instituto de Astrofísica de Andalucía (CSIC) and with the $2.1 \mathrm{~m}$ Otto Struve Telescope at McDonald Observatory of the University of Texas at Austin (USA).
}

are recognized by the presence of emission lines in one or both components, present the most challenging case and need to be studied on the individual basis. To obtain an accurate orbital solution and to study the behavior of activity, one needs to separate radial velocities, equivalent widths (EW hereafter) of emission lines, and fluxes between two components. It is also vital to carry out a precise stellar classification due to the importance of the subgiant and giant presence in the activity-rotation relation (e.g., in the RS CVn case).

Here, we concentrate on two recently discovered, X-ray/EUVselected, chromospherically active double-line spectroscopic binaries included in this survey: V789 Mon (2RE J0725-002, BD-00 1712) and GZ Leo (2RE J1101+223, HD 95559, BD+23 2297, HIP 53923).

V789 Mon, $V=9.33$, was classified by Jeffries et al. (1995) as a double-line spectroscopic binary (SB2) with almost identical K5V components. They gave a rotational velocity, $v \sin i$, of $25 \mathrm{~km} \mathrm{~s}^{-1}$ and an orbital period of 1.40 days. The photometric period (1.412 days) reported by Cutispoto et al. (1999) and Robb \& Gladders (1996) indicates synchronous rotation. Jeffries et al. (1995) suggest that this system could be eclipsing, but Robb \& Gladders (1996) did not find any evidence of eclipses in their photometric observations. Cutispoto et al. (1999) derived a K3V + M0:V classification for this system from colors and spectral signatures. Regarding activity, Jeffries et al. (1995) detected the $\mathrm{H} \alpha$ line in emission above the continuum level for both components.

GZ Leo is an SB2 binary with $V=8.8$ mag. Jeffries et al. (1995) obtained a first orbital solution with a period of 1.528 days using 11 radial velocity measurements. Later, Fekel \& Henry (2000) reported an improved orbital solution using nine new radial velocity determinations and one additional value given by Strassmeier et al. (2000). The system consists of two similar rapidly rotating stars $\left(v \sin i \approx 30 \mathrm{~km} \mathrm{~s}^{-1}\right)$. The spectral type of the components is not well established. Jeffries et al. 
Table 1

Observing Runs

\begin{tabular}{|c|c|c|c|c|c|c|c|}
\hline Date & Telescope & Instrument & CCD chip & $\begin{array}{c}\text { Spect. range } \\
(\AA)\end{array}$ & Orders & $\begin{array}{c}\text { Dispersion } \\
\text { (̊̊/pixel) }\end{array}$ & $\begin{array}{c}\text { FWHM } \\
(\AA)\end{array}$ \\
\hline 1998 Jan $12-21$ & $2.1 \mathrm{~m} \mathrm{OS}^{\mathrm{a}}$ & Sandiford & $1200 \times 400 \mathrm{CCD}$ & $6400-8800$ & 32 & $0.06-0.08$ & $0.13-0.20$ \\
\hline
\end{tabular}

Notes.

a $2.1 \mathrm{~m}$ Otto Struve Telescope at McDonald Observatory (Texas, USA).

b $2.2 \mathrm{~m}$ Telescope at the German Spanish Astronomical Observatory (CAHA) (Almería, Spain).

Table 2

Stellar Parameters

\begin{tabular}{|c|c|c|c|c|c|c|c|c|c|c|}
\hline Name & $T_{\mathrm{sp}}$ & SB & $B-V$ & $V$ & $\begin{array}{c}P_{\text {orb }}{ }^{\mathrm{a}} \\
\text { (days) }\end{array}$ & $\begin{array}{l}P_{\text {phot }} \\
\text { (days) }\end{array}$ & $\begin{array}{c}v \sin i^{\mathrm{a}} \\
\left(\mathrm{km} \mathrm{s}^{-1}\right)\end{array}$ & $\begin{array}{c}\pi \\
(\mathrm{mas})\end{array}$ & $\begin{array}{c}\mu_{\alpha} \cos \delta \\
\left(\mathrm{mas}_{\mathrm{yr}}{ }^{-1}\right)\end{array}$ & $\begin{array}{c}\mu_{\delta} \\
\left(\operatorname{mas~yr}^{-1}\right)\end{array}$ \\
\hline V789 Mon & $\mathrm{K} 5 \mathrm{~V} / \mathrm{K} 5 \mathrm{~V}$ & 2 & 1.03 & 9.33 & 1.4021 & 1.412 & $28.28 \pm 1.59 / 25.09 \pm 2.06$ & $30.56 \pm 0.22^{\mathrm{a}}$ & $73.20 \pm 1.00$ & $-27.10 \pm 1.00$ \\
\hline GZ Leo & $\mathrm{K} 1 \mathrm{~V} / \mathrm{K} 1 \mathrm{~V}$ & 2 & 0.87 & 8.92 & 1.5260 & 1.526 & $26.23 \pm 1.13 / 26.92 \pm 1.14$ & $18.43 \pm 1.19$ & $-141.40 \pm 0.80$ & $5.30 \pm 0.80$ \\
\hline
\end{tabular}

Note. ${ }^{a}$ Values determined in this paper.

(1995) classified it as G5, while Popper (1996) as K2, based on the strength of the $\mathrm{Na} \mathrm{I}_{1} \& \mathrm{D}_{2}$ lines. The latter is closer to the K1V type determined by Fekel \& Henry (2000) from the temperature and luminosity-sensitive line ratios in the 6430 $6465 \AA$ A region. Popper (1996) reported photometric variability, but could not confirm whether it is due to eclipses. Strassmeier et al. (2000) determined a period of 2.944 days from their photometric observations, but noted that a period of 1.514 days was also possible. Finally, Fekel \& Henry (2000) and Pandey et al. $(2002,2005)$ confirmed that the photometric period (1.526 days) is nearly identical to its orbital period. This binary system shows the typical signatures of chromospheric activity. A filledin $\mathrm{H} \alpha$ absorption line in both components was reported by Jeffries et al. (1995), while Ca II H \& K emissions were detected in the spectra by Strassmeier et al. (2000). In addition, GZ Leo is a young system as indicated by its large lithium abundance (Strassmeier et al. 2000; Fekel \& Henry 2000).

A preliminary analysis of these two systems can be found in Gálvez et al. (2003) and Gálvez (2005).

In Section 2, we summarize our observations. In Section 3, we derive stellar parameters and the orbit elements of the systems. The behavior of the different chromospheric activity indicators is described in Section 4. Finally, Section 5 gives a summary.

\section{OBSERVATIONS}

In this paper, we have analyzed high-resolution echelle spectra of both systems that we obtained during two observing runs detailed in Table 1 . The spectra were extracted using the standard reduction procedures in the $\mathrm{IRAF}^{3}$ echelle package (bias subtraction, flat-field division and optimal extraction of the spectra). We obtained the wavelength calibration by taking spectra of a Th-Ar lamp. Finally, we normalized the spectra by a polynomial fit to the observed continuum.

We obtained nine spectra of V789 Mon in one observing run and 15 spectra of GZ Leo in two. The signal-to-noise ratio $(\mathrm{S} / \mathrm{N})$ achieved in the $\mathrm{H} \alpha$ line region in all the spectra is $\approx 60$ for V789 Mon and $\approx 100$ for GZ Leo.

\footnotetext{
3 IRAF is distributed by the National Optical Observatory, which is operated by the Association of Universities for Research in Astronomy, Inc., under contract with the National Science Foundation.
}

\section{STELLAR PARAMETERS OF THE BINARY SYSTEMS}

We list the adopted stellar parameters of both systems in Table 2. Spectral type and the photometric data $(B-V, V$, $\left.P_{\text {phot }}\right)$ are taken from SIMBAD and Cutispoto et al. (1999) for V789 Mon and from SIMBAD and Fekel \& Henry (2000) for GZ Leo. The astrometric data (parallax, $\pi$; proper motions, $\mu_{\alpha} \cos \delta$ and $\mu_{\delta}$ ) are from Hipparcos (ESA 1997) and Tycho-2 (Høg et al. 2000) catalogues. For V789 Mon, no parallax value is available, so we give the spectroscopic parallax determined here (see Section 3.4). Finally, the orbital period $\left(P_{\text {orb }}\right)$ and the projected rotational velocities $(v \sin i)$ have been determined in this paper as well (see below).

\subsection{Spectral Types}

In order to obtain the spectral type of these binary systems, we compared our high-resolution echelle spectra with spectra of inactive reference stars of different spectral types and luminosity classes observed during the same observing run. To avoid contamination by chromospheric emission, we only used spectral orders that are free of lines sensitive to chromospheric activity. This analysis makes use of the program STARMOD developed at Penn State University (Barden 1985) and modified by us. STARMOD allows us to rotationally broaden spectra and shift them in the velocity space. We ran it on the spectra of the reference stars and combined with the appropriate weights to create composite spectra that were used as template for comparison with our targets.

For V789 Mon, we obtained the best fit between observed and synthetic spectra using a K5V reference star (HR 1614) for both components, contributing, respectively, $60 \%$ and $40 \%$ of the total flux in the continuum. The fits were carried out in all the phases where the spectra of the components could be separated. Our spectral classification is in agreement with a K5V type reported by Jeffries et al. (1995), while the relative contribution of the companions is compatible with Cutispoto et al. (1999) result that assigned a later spectral type for the secondary component.

In the case of GZ Leo, we obtained the best fit between observed and synthetic spectra, using a K0V reference star (HR 166) for both components, with the equal contribution from each component. Similar to V789 Mon, the fits were performed in all the phases where the components could be separated, and 
several spectral types and luminosity class reference stars were used. Our spectral classification is very close to the K2V type reported by Popper (1996) and the K1V type by Fekel \& Henry (2000).

\subsection{Rotational Velocity}

Jeffries et al. (1995) estimated the projected rotational velocity $(v \sin i)$ for $\mathrm{V} 789$ Mon as $25 \mathrm{~km} \mathrm{~s}^{-1}$. For GZ Leo, they found $27 \mathrm{~km} \mathrm{~s}^{-1}$, while Fekel \& Henry (2000) reported values of 32.4 and $31.6 \mathrm{~km} \mathrm{~s}^{-1}$ for each component. Fekel \& Henry (2000) noted that the actual $v \sin i$ may slightly smaller, because spots cause the lines to be shallower than in an unspotted star, making FWHM appear larger. Strassmeier et al. (2000) reported similar values of 31 and $26 \mathrm{~km} \mathrm{~s}^{-1}$, respectively.

Using STARMOD, we obtained for each observing run $v \sin i$ values about $30 \mathrm{~km} \mathrm{~s}^{-1}$ for both components of V789 Mon and GZ Leo. In order to determine a more accurate rotational velocities we applied a cross-correlation technique to our highresolution echelle spectra of these stars using IRAF task FXCOR. The method is described in detail in our previous papers (see Gálvez et al. 2002; López-Santiago et al. 2003). In short, the method is based on the fact that when a stellar spectrum with rotationally broadened lines is cross correlated against a narrowline spectrum, the width of the cross-correlation function $(\mathrm{CCF})$ will depend on the amount of rotational broadening of the former spectrum.

For V789 Mon, we used as a template a slowly rotating K5V star HR 1614. We obtained a $v \sin i$ of $28.28 \pm 1.59$ and 25.09 $\pm 2.06 \mathrm{~km} \mathrm{~s}^{-1}$ for the primary and the secondary components, respectively.

In the case of GZ Leo, we used a K0V star HR 166 and a K2V star HD 166620 as templates, in McDonald and FOCES observing runs, respectively. We obtained averaged values of $26.23 \pm 1.13$ and $26.92 \pm 1.14 \mathrm{~km} \mathrm{~s}^{-1}$ for each component (see Table 2).

\subsection{Radial Velocities and Orbital Solution}

Heliocentric radial velocities were also obtained by using the cross-correlation technique (see, e.g., Gálvez et al. 2007). The spectra of the target were cross-correlated order by order, using the routine FXCOR in IRAF, against spectra of radial velocity standards with similar spectral type taken from Beavers et al. (1979). We derived the radial velocity for each order from the position of peak of the CCF and calculated the uncertainties based on the fitted peak height and the antisymmetric noise as described by Tonry \& Davis (1979).

Since both systems are SB2, we could see two peaks in the CCF associated with two components; we fitted each peak separately. When the component peaks were too close, we used deblending technique.

In Tables 3 and 4, we list, for each spectrum, the heliocentric radial velocities $\left(V_{\text {hel }}\right)$ and their associated errors $\left(\sigma_{V}\right)$. The latter are obtained as weighted means of the individual values deduced for each order in V789 Mon and GZ Leo spectra. We note that the uncertainties returned by FXCOR for SB2 binaries are overestimated; when fitting each component, the presence of the other component will increase the antisymmetric noise, thereby biasing the error.

To compute the orbital solution for these systems, we combined our radial velocity data (for both components) with the data of Jeffries et al. (1995), Fekel \& Henry (2000), and Strassmeier et al. (2000); see Tables 3 and 4. The radial velocity
Table 3

Radial Velocities of V789 Mon

\begin{tabular}{|c|c|c|c|c|}
\hline Obs. & $\begin{array}{c}\text { HJD } \\
2400000+\end{array}$ & $\begin{array}{c}\mathrm{S} / \mathrm{N} \\
(\mathrm{H} \alpha)\end{array}$ & $\begin{array}{c}\text { Primary } \\
V_{\text {hel }} \pm \sigma_{V} \\
\left(\mathrm{~km} \mathrm{~s}^{-1}\right)\end{array}$ & $\begin{array}{c}\text { Secondary } \\
V_{\text {hel }} \pm \sigma_{V} \\
\left(\mathrm{~km} \mathrm{~s}^{-1}\right)\end{array}$ \\
\hline $\operatorname{JEF}(95) *$ & 49054.400 & $\ldots$ & $57.8 \pm 2.0$ & $-46.1 \pm 2.0$ \\
\hline $\operatorname{JEF}(95) *$ & 49054.444 & $\ldots$ & $71.9 \pm 2.0$ & $-63.6 \pm 2.0$ \\
\hline $\mathrm{JEF}(95) *$ & 49054.456 & $\ldots$ & $81.6 \pm 2.0$ & $-69.8 \pm 2.0$ \\
\hline $\operatorname{JEF}(95) *$ & 49054.507 & $\ldots$ & $96.3 \pm 2.0$ & $-84.6 \pm 2.0$ \\
\hline $\operatorname{JEF}(95) *$ & 49054.555 & $\ldots$ & $103.2 \pm 2.0$ & $-92.2 \pm 2.0$ \\
\hline $\mathrm{JEF}(95) *$ & 49055.394 & $\ldots$ & $-89.6 \pm 2.0$ & $109.2 \pm 2.0$ \\
\hline $\operatorname{JEF}(95) *$ & 49055.451 & $\ldots$ & $-79.5 \pm 2.0$ & $102.6 \pm 2.0$ \\
\hline $\operatorname{JEF}(95) *$ & 49055.500 & $\cdots$ & $-69.1 \pm 2.0$ & $85.4 \pm 2.0$ \\
\hline $\operatorname{JEF}(95) *$ & 49056.449 & $\cdots$ & $-21.2 \pm 2.0$ & $32.4 \pm 2.0$ \\
\hline $\operatorname{JEF}(95) *$ & 49056.496 & $\ldots$ & $-40.3 \pm 2.0$ & $54.3 \pm 2.0$ \\
\hline $\operatorname{JEF}(95) *$ & 49056.572 & $\ldots$ & $-68.1 \pm 2.0$ & $91.1 \pm 2.0$ \\
\hline MCD98 & 50826.835 & 152 & $105.40 \pm 0.66$ & $-100.66 \pm 0.88$ \\
\hline MCD98 & 50828.837 & 76 & $-80.61 \pm 0.66$ & $93.65 \pm 0.93$ \\
\hline MCD98 & 50829.854 & 70 & $74.97 \pm 0.76$ & $-63.90 \pm 1.38$ \\
\hline MCD98 & 50830.848 & 59 & $61.17 \pm 0.67$ & $-51.52 \pm 0.86$ \\
\hline MCD98 & 50831.870 & 46 & $-86.62 \pm 0.62$ & $104.17 \pm 0.95$ \\
\hline MCD98 & 50833.846 & 58 & $101.92 \pm 0.67$ & $-100.26 \pm 0.83$ \\
\hline MCD98 & 50834.879 & 56 & $-23.90 \pm 0.62$ & $33.66 \pm 0.89$ \\
\hline MCD98 & 50835.844 & 47 & $-72.60 \pm 0.66$ & $85.18 \pm 1.00$ \\
\hline MCD98 & 50832.843 & 99 & $2.87 \pm 4.28$ & $6.44 \pm 4.00$ \\
\hline
\end{tabular}

Note. * JEF(95): Jeffries et al. (1995).

data are plotted in Figure 1 for V789 Mon and GZ Leo on the leftand right-hand sides, respectively. Solid symbols represent the primary, and open symbols represent the secondary. The curves represent a minimum $\chi^{2}$ fit orbit solution. The orbit-fitting code uses the Numerical Recipes (Press et al. 1986) implementation of the Levenberg-Marquardt method of fitting a nonlinear function to the data and weights each datum according to its associated uncertainty (see, e.g., Gálvez et al. 2002, 2007 for further details).

We found a nearly circular $(e=0.0129)$ for V789 Mon system, with an orbital period of 1.4021 days which confirms it as a synchronous system $\left(P_{\text {phot }} \approx 1.412\right.$ days, Cutispoto et al. 1999). The mass ratio $(q=1.0542)$ indicates that both components are very similar. The obtained parameters are in agreement with Jeffries et al. (1995); see Table 5 for details.

We arrived at a similar conclusions for GZ Leo: a nearly circular orbit ( $e=0.0073$ ) with an orbital period of 1.5260 days, which is very similar to the photometric rotational period $\left(P_{\text {phot }}\right.$ $\approx 1.5264$ days, Fekel \& Henry 2000), indicating a synchronized rotation. The obtained minimum masses $\left(M \sin ^{3} i\right)$ and the mass ratio $(q=1.0139)$ are in agreement with Fekel \& Henry (2000); see Table 6 for computed parameters.

\subsection{Physical Parameters}

Adopting spectral types derived in Section 3.1 for the primary components and adopting masses and temperatures from Landolt-Börnstein tables (Schmidt-Kaler 1982), we derived masses and spectral types for the secondaries.

We also estimated minimum radii $(R \sin i)$, luminosities and minimum masses ( $M \sin i$ ) of the components using photometric periods (from the literature, see Section 3) and rotational velocities (from Section 3.2). The results, given in Table 7 , confirm our previous classification.

Furthermore, since Hipparcos parallax of V789 Mon is not known, we calculated a spectroscopic parallax. Using bolometric magnitude of a $\mathrm{K} 5 \mathrm{~V}$ star $(+6.7)$, the bolometric 

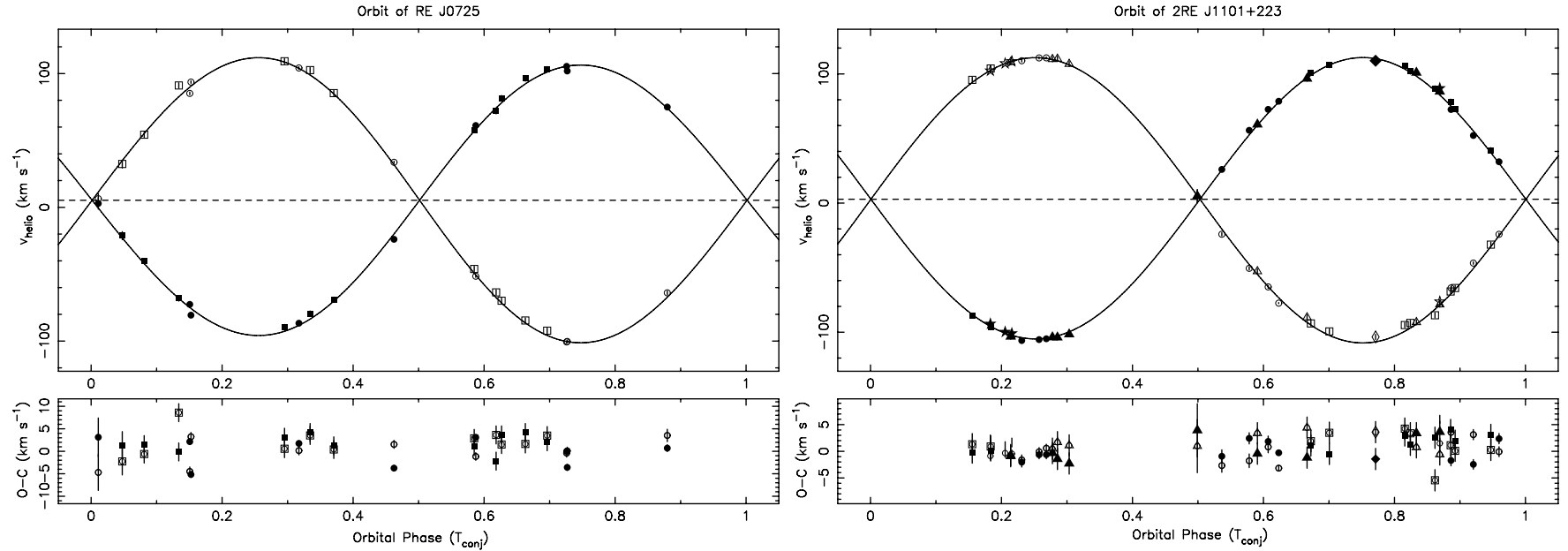

Figure 1. Radial velocity data and fit vs. the orbital phase. Solid symbols represent the primary and open symbols represent the secondary. V789 Mon (2RE J0725-002) on the left (squares data from Jeffries et al. 1995 and circles data from our observations) and GZ Leo (2RE J1101+223) on the right (squares data from Jeffries et al. 1995, triangles from Fekel \& Henry 2000, rhombus from Strassmeier et al. 2000, circles from our McDonald98 observing run and stars from our FOCES02 observing run). The solid curves represent a minimum $\chi^{2}$ fit orbit solution as described in the text.

Table 4

Radial Velocities of GZ Leo

\begin{tabular}{|c|c|c|c|c|}
\hline Obs. & $\begin{array}{c}\text { HJD } \\
2400000+\end{array}$ & $\begin{array}{l}\mathrm{S} / \mathrm{N} \\
(\mathrm{H} \alpha)\end{array}$ & $\begin{array}{l}\text { Primary } \\
V_{\text {hel }} \pm \sigma_{V} \\
\left(\mathrm{~km} \mathrm{~s}^{-1}\right)\end{array}$ & $\begin{array}{c}\text { Secondary } \\
V_{\text {hel }} \pm \sigma_{V} \\
\left(\mathrm{~km} \mathrm{~s}^{-1}\right)\end{array}$ \\
\hline $\mathrm{JEF}(95)^{\mathrm{a}}$ & 49052.571 & $\ldots$ & $101.9 \pm 2.0$ & $-92.8 \pm 2.0$ \\
\hline $\operatorname{JEF}(95)^{\mathrm{a}}$ & 49052.664 & $\ldots$ & $78.5 \pm 2.0$ & $-68.5 \pm 2.0$ \\
\hline $\operatorname{JEF}(95)^{\mathrm{a}}$ & 49052.757 & $\ldots$ & $40.9 \pm 2.0$ & $-32.2 \pm 2.0$ \\
\hline $\operatorname{JEF}(95)^{\mathrm{a}}$ & 49054.600 & $\ldots$ & $-87.2 \pm 2.0$ & $95.4 \pm 2.0$ \\
\hline $\operatorname{JEF}(95)^{\mathrm{a}}$ & 49054.644 & $\ldots$ & $-95.9 \pm 2.0$ & $104.2 \pm 2.0$ \\
\hline $\operatorname{JEF}(95)^{\mathrm{a}}$ & 49055.389 & $\ldots$ & $100.7 \pm 2.0$ & $-93.2 \pm 2.0$ \\
\hline $\operatorname{JEF}(95)^{\mathrm{a}}$ & 49055.435 & $\ldots$ & $106.8 \pm 2.0$ & $-99.4 \pm 2.0$ \\
\hline $\operatorname{JEF}(95)^{\mathrm{a}}$ & 49055.611 & $\ldots$ & $106.2 \pm 2.0$ & $-94.5 \pm 2.0$ \\
\hline $\operatorname{JEF}(95)^{\mathrm{a}}$ & 49055.679 & $\ldots$ & $88.7 \pm 2.0$ & $-86.9 \pm 2.0$ \\
\hline $\operatorname{JEF}(95)^{\mathrm{a}}$ & 49055.727 & $\ldots$ & $72.8 \pm 2.0$ & $-65.8 \pm 2.0$ \\
\hline $\operatorname{FEKEL}(00)^{\mathrm{b}}$ & 50200.709 & $\ldots$ & $-103.4 \pm 2.0$ & $109.1 \pm 2.0$ \\
\hline $\operatorname{FEKEL}(00)^{\mathrm{b}}$ & 50400.035 & $\ldots$ & $101.0 \pm 2.0$ & $-92.3 \pm 2.0$ \\
\hline FEKEL $(00)^{\mathrm{b}}$ & 50401.049 & $\ldots$ & $5.4 \pm 2.0$ & $5.4 \pm 2.0$ \\
\hline $\operatorname{FEKEL}(00)^{\mathrm{b}}$ & 50576.681 & $\ldots$ & $60.9 \pm 2.0$ & $-52.9 \pm 2.0$ \\
\hline $\operatorname{FEKEL}(00)^{\mathrm{b}}$ & 50632.664 & $\ldots$ & $-103.9 \pm 2.0$ & $111.4 \pm 2.0$ \\
\hline FEKEL $(00)^{\mathrm{b}}$ & 50831.054 & $\ldots$ & $-104.0 \pm 2.0$ & $111.6 \pm 2.0$ \\
\hline FEKEL $(00)^{\mathrm{b}}$ & 50831.947 & $\ldots$ & $86.4 \pm 2.0$ & $-78.6 \pm 2.0$ \\
\hline FEKEL $(00)^{\mathrm{b}}$ & 50927.773 & $\ldots$ & $96.4 \pm 2.0$ & $-88.6 \pm 2.0$ \\
\hline MCD98 & 50826.992 & 123 & $-77.48 \pm 0.44$ & $78.84 \pm 0.16$ \\
\hline MCD98 & 50827.975 & 133 & $112.42 \pm 0.75$ & $-105.11 \pm 0.78$ \\
\hline MCD98 & 50829.032 & 123 & $-24.19 \pm 0.93$ & $31.98 \pm 0.96$ \\
\hline MCD98 & 50830.019 & 116 & $-64.94 \pm 1.10$ & $72.53 \pm 1.02$ \\
\hline MCD98 & 50831.010 & 86 & $112.36 \pm 0.77$ & $-105.72 \pm 0.76$ \\
\hline MCD98 & 50832.024 & 92 & $-46.47 \pm 0.96$ & $52.29 \pm 0.90$ \\
\hline MCD98 & 50833.027 & 132 & $-50.52 \pm 1.28$ & $56.33 \pm 1.05$ \\
\hline MCD98 & 50834.024 & 100 & $110.18 \pm 0.92$ & $-106.47 \pm 0.92$ \\
\hline MCD98 & 50835.025 & 80 & $-65.73 \pm 1.12$ & $72.44 \pm 1.01$ \\
\hline MCD98 & 50836.017 & 97 & $-24.11 \pm 1.26$ & $26.04 \pm 1.20$ \\
\hline FEKEL $(00)^{\mathrm{b}}$ & 51240.763 & $\ldots$ & $110.2 \pm 2.0$ & $-103.7 \pm 2.0$ \\
\hline $\operatorname{STRAS}(00)^{\mathrm{c}}$ & 51659.696 & $\ldots$ & $-101.6 \pm 2.0$ & $107.7 \pm 2.0$ \\
\hline FOCES02 & 52387.409 & 77 & $-93.69 \pm 0.75$ & $102.35 \pm 0.78$ \\
\hline FOCESO2 & 52388.458 & 82 & $88.72 \pm 0.76$ & $-76.47 \pm 0.96$ \\
\hline FOCESO2 & 52389.460 & 97 & $4.76 \pm 0.24$ & $4.76 \pm 0.24$ \\
\hline FOCESO2 & 52390.496 & 96 & $-99.96 \pm 0.69$ & $108.04 \pm 0.79$ \\
\hline FOCES02 & 52390.512 & 68 & $-101.09 \pm 082$ & $109.61 \pm 0.68$ \\
\hline
\end{tabular}

Notes.

a JEF(95): Jeffries et al. (1995).

b FEKEL(00): Fekel \& Henry (2000).

${ }^{c}$ STRAS(00): Strassmeier et al. (2000).
Table 5

Orbital Solution of V789 Mon

\begin{tabular}{lccc}
\hline \hline Element & Value & Uncertainty & Units \\
\hline$P_{\text {orb }}$ & 1.4021 & 0.0000 & days \\
$T_{\text {conj }}$ & 49054.980 & 0.048 & HJD \\
$\omega$ & 269.44 & 0.23 & degrees \\
$e$ & 0.0129 & 0.0024 & \\
$K_{\mathrm{P}}$ & 101.09 & 0.47 & $\mathrm{~km} \mathrm{~s}^{-1}$ \\
$K_{\mathrm{S}}$ & 106.57 & 0.69 & $\mathrm{~km} \mathrm{~s}^{-1}$ \\
$\gamma$ & 5.29 & 0.17 & $\mathrm{~km} \mathrm{~s}^{-1}$ \\
$q=M_{\mathrm{P}} / M_{\mathrm{S}}$ & 1.0542 & 0.0047 & \\
$a_{\mathrm{P}} \sin i$ & 1.9490 & 0.0092 & $10^{6} \mathrm{~km}$ \\
$a_{\mathrm{S}} \sin i$ & 2.054 & 0.013 & $10^{6} \mathrm{~km}$ \\
$a \operatorname{sen} i$ & 4.003 & 0.016 & $10^{6} \mathrm{~km}$ \\
$"$ & 0.027 & & $\mathrm{AU}$ \\
, & 5.752 & & $\mathrm{R}_{\odot}$ \\
$M_{\mathrm{P}} \sin ^{3} i$ & 0.6675 & 0.0085 & $M_{\odot}$ \\
$M_{\mathrm{S} \sin }{ }^{3} i$ & 0.6332 & 0.0081 & $M_{\odot}$ \\
$\mathrm{f}(M)$ & 0.1500 & 0.0021 & $M_{\odot}$ \\
\hline
\end{tabular}

correction $\left(B C=-0^{\mathrm{m}} 72\right)$ corresponding to a $\mathrm{K} 5 \mathrm{~V}$, and the luminosity ratio, we obtain the bolometric and the visual total absolute magnitudes for the system. Comparing the total visual absolute magnitude (6.76), with the measured $\mathrm{V}$ magnitude (9.33), we derive a distance of $32.72 \pm 0.13 \mathrm{pc}(\pi=30.56 \pm$ 0.22 mas) for V789 Mon. Due to possible activity-induced variations in the $\mathrm{V}$, we expect a $10 \%$ additional error in this distance measure. Since the system is relatively close, the interstellar reddening is neglected.

Using the total luminosity based on the measured distance $(54.26 \mathrm{pc})$ for GZ Leo, we obtained the total magnitude for the system $V=8.77$. The difference between the observed $V$ magnitude (8.92) and the calculated, one can be explained by the presence of photospheric cool spots over the stellar surfaces of both components.

\subsection{Kinematics}

We computed the galactic space-velocity components $(U, V$, $W$ ) of these systems using as radial velocity the center of mass velocity $(\gamma)$ and accurate proper motions and parallax taken from Hipparcos (ESA 1997) and Tycho-2 (Høg et al. 2000) 

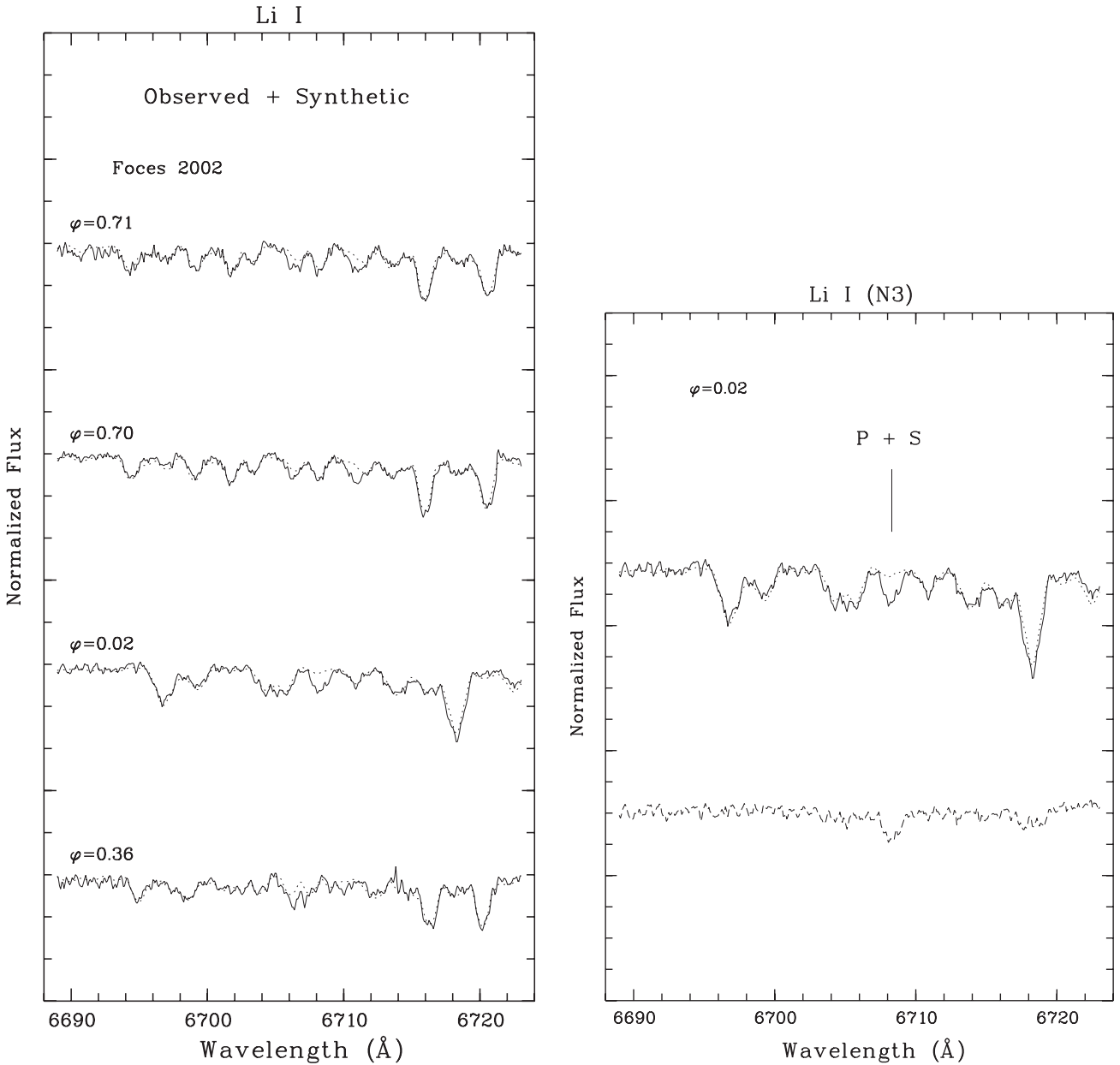

Figure 2. Left: spectra in different phases of GZ Leo in the Li I line region. The observed (solid-line) and the synthesized spectrum (dashed-line) show the lithium absortion. Right: the conjunction phase where we measured the EW(Li I). The observed and the synthesized spectrum are plotted at the top and the subtracted spectrum (dotted line) at the bottom.

Table 6

Orbital Solution of GZ Leo

\begin{tabular}{lccc}
\hline \hline Element & Value & Uncertainty & Units \\
\hline$P_{\text {orb }}$ & 1.5260 & 0.0000 & days \\
$T_{\text {conj }}$ & 49051.312 & 0.080 & HJD \\
$\omega$ & 359.33 & 18.98 & degrees \\
$e$ & 0.0073 & 0.0025 & \\
$K_{\mathrm{P}}$ & 108.92 & 0.48 & $\mathrm{~km} \mathrm{~s}^{-1}$ \\
$K_{\mathrm{S}}$ & 110.43 & 0.58 & $\mathrm{~km} \mathrm{~s}^{-1}$ \\
$\gamma$ & 2.93 & 0.12 & $\mathrm{~km} \mathrm{~s}^{-1}$ \\
$q=M_{\mathrm{P}} / M_{\mathrm{S}}$ & 1.0139 & 0.0029 & \\
$a_{\mathrm{P}} \sin i$ & 2.285 & 0.010 & $10^{6} \mathrm{~km}$ \\
$a_{\mathrm{S}} \sin i$ & 2.317 & 0.012 & $10^{6} \mathrm{~km}$ \\
$a \operatorname{sen} i$ & 4.603 & 0.016 & $10^{6} \mathrm{~km}$ \\
$”$ & 0.031 & & $\mathrm{AU}$ \\
, & 6.613 & & $\mathrm{R}_{\odot}$ \\
$M_{\mathrm{P}} \sin ^{3} i$ & 0.8400 & 0.0091 & $M_{\odot}$ \\
$M_{\mathrm{S}} \sin ^{3} i$ & 0.8285 & 0.0090 & $M_{\odot}$ \\
$\mathrm{f}(M)$ & 0.2043 & 0.0027 & $M_{\odot}$ \\
\hline
\end{tabular}

catalogues (see Table 2), except for spectroscopic parallax of V789 Mon that has been calculated here.

The obtained values and associated errors are given in Table 8 . The velocity components lie in the $(U, V)$ diagram obviously inside the young disk population boundaries (Eggen 1984, 1989; Montes et al. 2001a, 2001b) indicating that both systems are young disk stars. In addition, the Eggen kinematic criteria predict that GZ Leo may be a member of Hyades supercluster (see Montes et al. 2001b and Gálvez 2005).

\subsection{The Li I $\lambda 6707.8$ Line}

The resonance doublet of $\mathrm{Li}$ I at $\lambda 6707.8 \AA$ is a spectroscopic feature very important in the diagnostic of age in late-type stars, since it is destroyed easily by thermonuclear reactions in the stellar interior.

The spectral region of this line is included in all our spectra for both systems.

At the spectral resolution of our spectra and with the rotational velocity, we have determined for the components of both binaries, the Li I line is blended with the nearby Fe $\mathrm{I} \lambda 6707.41 \AA$ line. In the case of V789 Mon the Li I line is very weak, and we could not measure the EW with the required precision. For GZ Leo, we clearly see the $\mathrm{Li}$ I+Fe I absorption feature in all the spectra (see Figure 2, left side), but due to the blending with other photospheric lines of both components, we have only measured the EW in the spectrum of the third night of the FOCES02 observing run (see Figure 2, right) which is very close to conjunction $(\varphi=0.02)$. The obtained value $\mathrm{EW}$ $(\mathrm{Li} \mathrm{I}+\mathrm{Fe} \mathrm{I})=60.7 \mathrm{m \AA}$ contain the contribution from both components, but taken into account that they have the same spectral type and contribution to the continuum we can assume that this is also the $\mathrm{EW}(\mathrm{Li} \mathrm{I}+\mathrm{Fe} \mathrm{I})$ of each component. We have corrected this total EW by subtracting the EW $(\mathrm{Fe} \mathrm{I})=$ 

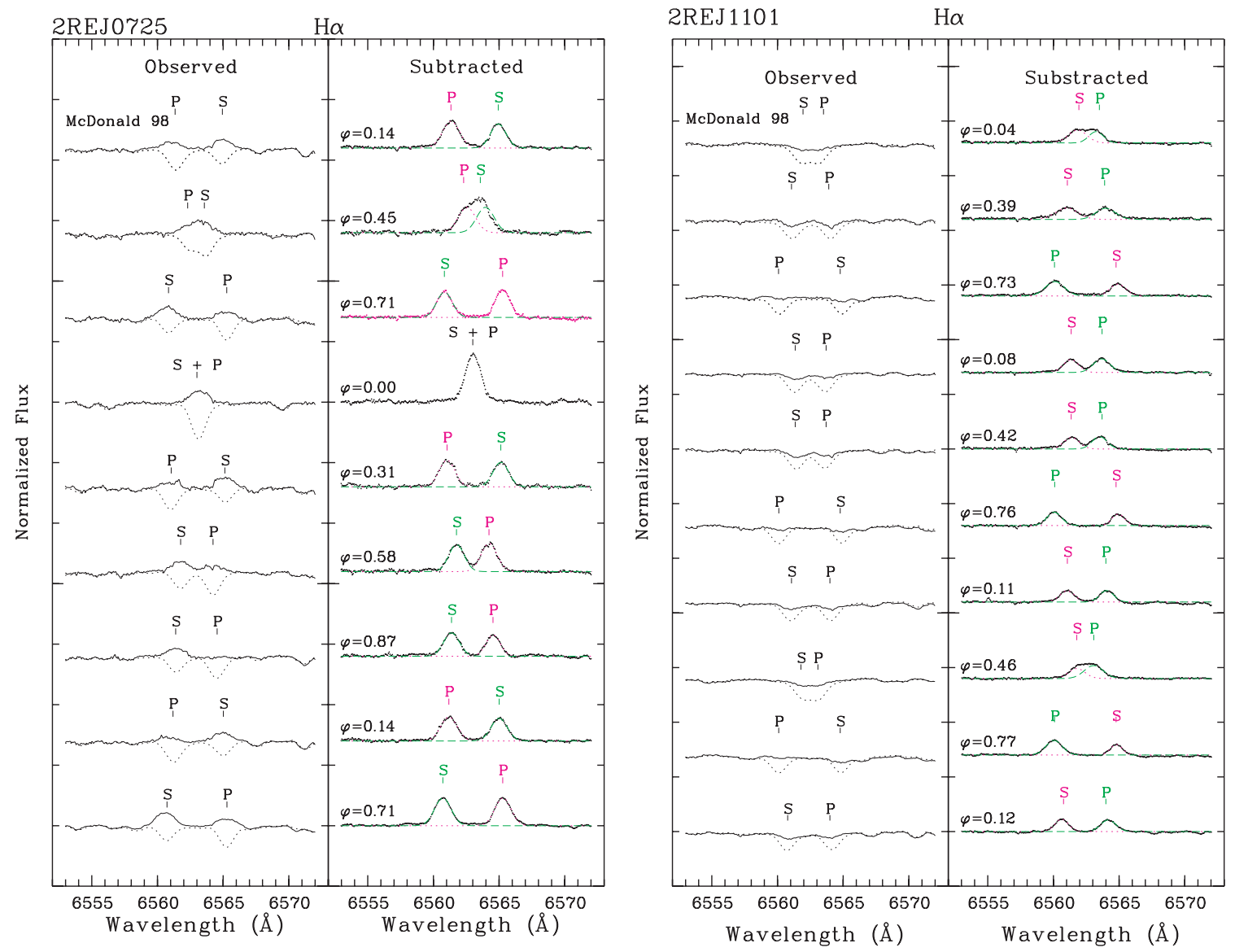

Figure 3. Left: spectra of V789 Mon (2RE J0725) in the H $\alpha$ line region in McDonald run. Right: spectra of GZ Leo (2RE J1101) in the H $\alpha$ line region in McDonald run. The observed spectrum (solid-line) and the synthesized spectrum (dashed-line) are plotted in the left panel and the subtracted spectrum (dotted line) in the right panel. The position of the $\mathrm{H} \alpha$ line for the primary (P) and secondary (S) components are marked too and here we again have the two-Gaussian fit superposed.

(A color version of this figure is available in the online journal.)

Table 7

Derived Stellar Parameters

\begin{tabular}{|c|c|c|c|c|c|c|c|c|c|c|c|}
\hline Name & $S p T_{\mathrm{P}}{ }^{\mathrm{a}}$ & $\begin{array}{c}M_{\mathrm{P}}^{\mathrm{a}} \\
\left(M_{\odot}\right) \\
\end{array}$ & $\begin{array}{c}M_{\mathrm{S}} \\
\left(M_{\odot}\right) \\
\end{array}$ & $S p T_{\mathrm{S}}{ }^{\mathrm{b}}$ & $\begin{array}{c}R \sin i_{\mathrm{P}} \\
\left(R_{\odot}\right)\end{array}$ & $\begin{array}{c}R \sin i_{\mathrm{S}} \\
\left(R_{\odot}\right)\end{array}$ & $\begin{array}{c}T_{\text {eff }}{ }^{\mathrm{a}} \\
(\mathrm{K}) \\
\end{array}$ & $\begin{array}{c}L_{\mathrm{P}} \\
\left(L_{\odot}\right) \\
\end{array}$ & $\begin{array}{c}L_{\mathrm{S}} \\
\left(L_{\odot}\right) \\
\end{array}$ & $\begin{array}{c}M_{\text {Pmin }} \\
\left(M_{\odot}\right) \\
\end{array}$ & $\begin{array}{c}M_{\text {Smin }} \\
\left(M_{\odot}\right) \\
\end{array}$ \\
\hline V789 Mon & K5V & 0.67 & 0.63 & K6-K7V & $0.79 \pm 0.03$ & $0.70 \pm 0.06$ & 4350 & 0.20 & 0.16 & 0.56 & 0.52 \\
\hline GZ Leo & K0V & 0.79 & 0.78 & $\mathrm{~K} 0 \mathrm{~V}$ & $0.79 \pm 0.03$ & $0.82 \pm 0.03$ & 5250 & 0.42 & 0.54 & 0.73 & 0.75 \\
\hline
\end{tabular}

Notes.

${ }^{a}$ From Landolt-Börnstein tables (Schmidt-Kaler 1982).

${ }^{\mathrm{b}}$ Derived.

$19.8 \mathrm{~m} \AA$ calculated from the empirical relationship with $(B-V)$ given by Favata et al. (1993) resulting EW (Li I) $=40.9 \mathrm{~m} \AA$. One additional estimation of the EW (Li I) in this spectrum can be obtained by using the spectral-subtraction technique. In the subtracted spectrum the contribution from the photospheric lines is eliminated, therefore the $\mathrm{EW}=51.2 \mathrm{~m} \AA$ measured in this spectrum is the total $\mathrm{EW}(\mathrm{Li} \mathrm{I})$ and, as both components are equal, also the EW(Li I) of each one. The difference in the EW obtained from both methods is likely due to the influence of the stellar metallicity in the calibration used in the first one to obtain the $\mathrm{EW}(\mathrm{Fe} \mathrm{I})$ and in the standard star used as reference in the subtraction technique that are not taken into account here. The EW(Li I) of each component we have determined ( $40.9 \mathrm{~m} \AA$ with the first method and $51.2 \mathrm{~m} \AA$ with the second one) is smaller than the $63 \mathrm{~m} \AA$ estimated by Fekel \& Henry (2000) and the 60 $m \AA$ given by Strassmeier et al. (2000). The small differences between these values can be attributed to the different method used by these authors to estimate the Fe I contribution and the error in the EW determination.

This small EW(Li I ) is in agreement with the values observed in Hyades cluster stars of this spectral type (see Montes et al. 2001a) and with the membership to the Hyades supercluster (see Section 3.5). That situated the system age in about $600 \mathrm{Myr}$.

\section{CHROMOSPHERIC ACTIVITY INDICATORS}

The echelle spectra analyzed in this work allowed us to study the behavior of the different chromospheric activity indicators, from the $\mathrm{Ca}$ II $\mathrm{H} \& \mathrm{~K}$ to the $\mathrm{Ca}$ II IRT lines, which are formed at different atmospheric heights. The chromospheric contribution in these features were determined by using the spectral-subtraction technique described in detail by Montes et al. $(1995,1997,1998,2000)$ and Gálvez et al. $(2002,2007)$.

The excess emission in EW is measured in the subtracted spectra, after correcting for the relative contribution of each 
Table 8

Galactic Space-Velocity Components

\begin{tabular}{lrrrr}
\hline \hline Name & $\begin{array}{c}U \pm \sigma_{U} \\
\left(\mathrm{~km} \mathrm{~s}^{-1}\right)\end{array}$ & $\begin{array}{c}V \pm \sigma_{V} \\
\left(\mathrm{~km} \mathrm{~s}^{-1}\right)\end{array}$ & $\begin{array}{c}W \pm \sigma_{W} \\
\left(\mathrm{~km} \mathrm{~s}^{-1}\right)\end{array}$ & $\begin{array}{c}V_{\text {Total }} \\
\left(\mathrm{km} \mathrm{s}^{-1}\right)\end{array}$ \\
\hline V789 Mon & $-32.78 \pm 2.07$ & $-10.67 \pm 0.70$ & $-12.10 \pm 0.96$ & 36.53 \\
GZ Leo & $0.33 \pm 0.46$ & $-7.92 \pm 0.49$ & $6.65 \pm 0.60$ & 10.37 \\
\hline
\end{tabular}

component in the total continuum. This contribution is determined by using the radii and temperatures adopted in Section 3. For instance, in the $\mathrm{H} \alpha$ line region the relative contributions were $S_{\mathrm{P}}=0.61$ for the primary component and $S_{\mathrm{S}}=0.39$ for the secondary component in V789 Mon and for GZ Leo we had the 0.50 contributions for both components.

In Table 9 we give the corrected EW for the $\mathrm{H} \alpha$ and $\mathrm{Ca}$ II IRT lines for V789 Mon. In Table 10 we list corrected EW for the Ca II $\mathrm{H} \& \mathrm{~K}, \mathrm{H} \epsilon, \mathrm{H} \delta, \mathrm{H} \gamma, \mathrm{H} \beta, \mathrm{H} \alpha$, and Ca II IRT ( $\lambda 8498$, $\lambda 8542, \lambda 8662 \AA$ ) lines for GZ Leo in FOCES run and for $\mathrm{H} \alpha$ and $\mathrm{Ca}$ II IRT in McDonald run. The corrected EWs is given separately for each component $(\mathrm{P} / \mathrm{S})$, unless the lines of both components overlap, in which case the combined $\mathrm{EW}$ is given. The final estimated errors in EW are in the range 10\%-20\% (see, e.g., Gálvez et al. 2007 for details).

Finally, corrected EWs were converted to the absolute surface fluxes by using the empirical stellar flux scales calibrated by Hall (1996) as a function of the star color index. In our case, we used the $B-V$ index and the corresponding coefficients for $\mathrm{Ca}$ II $\mathrm{H}$ $\& \mathrm{~K}, \mathrm{H} \alpha$ and $\mathrm{Ca}$ II IRT. For $\mathrm{H} \epsilon$, we used the same coefficients as for $\mathrm{Ca}$ II $\mathrm{H} \& \mathrm{~K}$, while for $\mathrm{H} \delta, \mathrm{H} \gamma$ and $\mathrm{H} \beta$ the coefficients were obtained by interpolating between the values for $\mathrm{Ca}$ II $\mathrm{H}$ $\& \mathrm{~K}$ and $\mathrm{H} \alpha$. In Tables 9 and 11, we give the logarithm of the calculated absolute flux at the stellar surface $\left(\log F_{\mathrm{S}}\right)$ for different chromospheric activity indicators.

Figures 3-5 show a representative regions around the $\mathrm{H} \alpha$, $\mathrm{Ca}$ II H \& K, and Ca II IRT $\lambda 8498, \lambda 8542$ lines. The observed (solid line) and the synthesized spectra (dashed line) are shown in the left panel, while subtracted spectrum (dotted line) in the right panel. The observing run and the orbital phase $(\varphi)$ of each spectrum are also shown in these figures. In Figure 6, we plot a representative subtracted spectra in $\mathrm{H} \beta, \mathrm{H} \gamma$ and $\mathrm{H} \delta$ region for GZ Leo.

\subsection{The Ho Line}

In V789 Mon, $\mathrm{H} \alpha$ is observed in emission above the continuum in all the observed spectra (see Figure 3, left panel of the left side). After applying the spectral-subtraction technique, one can see that $\mathrm{H} \alpha$ emission from the primary and secondary components looks very much alike.

In all the spectra, except one which is very close to conjunction, we measured the emission coming from both components by using a two-Gaussian fit to the subtracted spectra (see Figure 3, right panel of the left side). The combined EW is given when it was not possible to deblend the two components.

The persistence of $\mathrm{H} \alpha$ emission in V789 Mon spectra indicates that it is a very active binary system similar to some RS CVn and BY Dra systems, which always show $\mathrm{H} \alpha$ emission above the continuum. The EW measured in the subtracted spectra by Gaussian fitting gives an average value of $\operatorname{EW}(\mathrm{H} \alpha)$ $=0.65 / 0.62 \AA$ for primary and secondary components (which is higher than reported by Jeffries et al. (1995), $\mathrm{EW}(\mathrm{H} \alpha)=$ $0.09 / 0.25 \AA$ ).

In GZ Leo, we analyzed 15 spectra including the $\mathrm{H} \alpha$ line region. In the first run (McDonald98), the $\mathrm{H} \alpha$ line appears

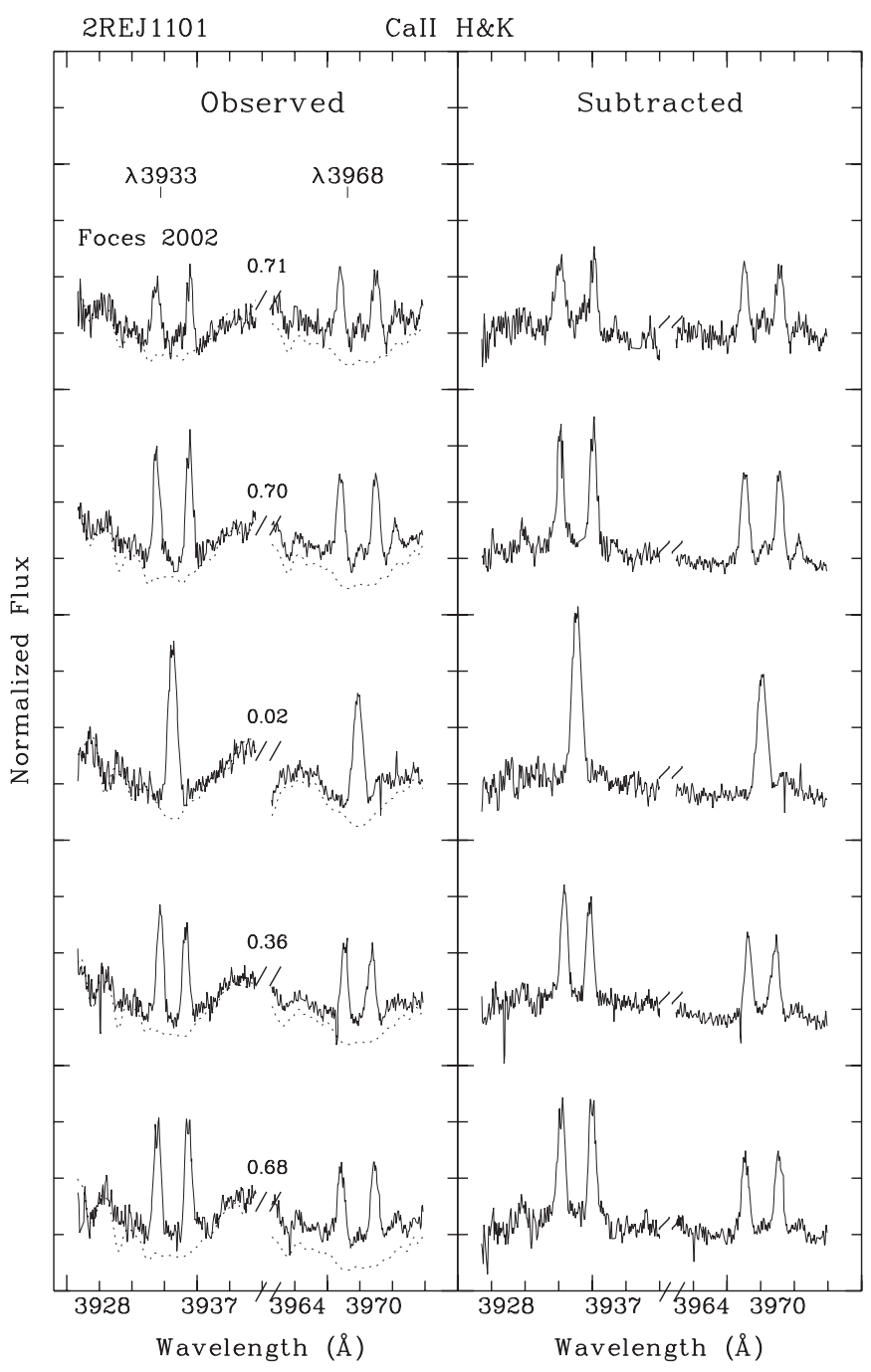

Figure 4. As in Figure 3, but in the region of the $\mathrm{Ca}$ II $\mathrm{H} \& \mathrm{~K}$ and $\mathrm{H} \epsilon$ lines for GZ Leo (2RE J1101) in FOCES run.

completely filled by emission in both components (see Figure 3 , left panel on the right side). As in the case of V789 Mon, the emission from both components is nearly identical and was measured by applying a two-Gaussian fit to the subtracted spectra (see Figure 3, right panel on the right side). The spectrum at phase 0.46 is close to conjunction, so the measured EW given in Table 10 is the combined EW. The mean values of EW are $\operatorname{EW}_{P}(\mathrm{H} \alpha)=0.32 \AA$ and $\mathrm{EW}_{S}(\mathrm{H} \alpha)=0.36 \AA$. We note however that a variation with phase is observed in both components, with a remarkable tendency to behave in the opposite way for Ca II IRT EWs; unfortunately the phase coverage is not enough for a reliable conclusion (Table 10).

When we analyze the FOCES02 run, we see again that the $\mathrm{H} \alpha$ line is completely filled-in by emission for both components. After fitting the subtracted spectra we measured the excess emission $\mathrm{EW}_{P}(\mathrm{H} \alpha)=0.46 \AA$ and $\mathrm{EW}_{S}(\mathrm{H} \alpha)=0.47 \AA$, i.e., a $35 \%$ increase compared to McDonald98 run.

In GZ Leo system the secondary star usually shows slightly larger values of $\mathrm{H} \alpha \mathrm{EW}$ and no variation with phase was detected (see Table 10).

\subsection{The $H \beta, H \gamma$ and $H \delta$ Lines}

The other three Balmer lines $(\mathrm{H} \beta, \mathrm{H} \gamma$ and $\mathrm{H} \delta$ ) were included only in some of our spectra for GZ Leo during the FOCES02. 
Table 9

EW and Absolute Surface Flux of the Different Chromospheric Activity Indicators of V789 Mon

\begin{tabular}{|c|c|c|c|c|c|c|c|c|}
\hline \multirow[t]{3}{*}{$\varphi$} & \multicolumn{4}{|c|}{ EW $(\AA ̊)$ (Primary/Secondary) } & \multicolumn{4}{|c|}{$\log F_{\mathrm{S}}$ (Primary/Secondary) } \\
\hline & \multirow[b]{2}{*}{$\mathrm{H} \alpha$} & \multicolumn{3}{|c|}{ Ca II IRT } & \multirow[b]{2}{*}{$\mathrm{H} \alpha$} & \multicolumn{3}{|c|}{ Ca II IRT } \\
\hline & & $\lambda 8498$ & $\lambda 8542$ & $\lambda 8662$ & & $\lambda 8498$ & $\lambda 8542$ & $\lambda 8662$ \\
\hline 0.71 & $0.71 / 0.72$ & $0.27 / 0.31$ & $0.42 / 0.29$ & $0.33 / 0.29$ & $6.83 / 7.02$ & $6.27 / 6.45$ & $6.46 / 6.42$ & $6.35 / 6.42$ \\
\hline 0.14 & $0.62 / 0.58$ & $0.19 / 0.22$ & $0.33 / 0.53$ & $0.13 / 0.36$ & $6.77 / 6.93$ & $6.11 / 6.23$ & $6.35 / 6.68$ & $5.95 / 6.51$ \\
\hline 0.87 & $0.48 / 0.58$ & $\cdots$ & $\cdots$ & $\cdots$ & $6.66 / 6.93$ & $\cdots$ & $\cdots$ & $\cdots$ \\
\hline 0.58 & $0.70 / 0.65$ & $0.30 / 0.25$ & $0.34 / 0.39$ & $0.34 / 0.28$ & $6.82 / 6.98$ & $6.31 / 6.35$ & $6.36 / 6.55$ & $6.36 / 6.40$ \\
\hline 0.31 & $0.66 / 0.60$ & $0.35 / 0.34$ & $0.32 / 0.33$ & $0.38 / 0.37$ & $6.80 / 6.94$ & $6.38 / 6.49$ & $6.34 / 6.47$ & $6.41 / 6.52$ \\
\hline 0.00 & $1.24^{\mathrm{a}}$ & $0.39^{\mathrm{a}}$ & $0.63^{\mathrm{a}}$ & $0.52^{\mathrm{a}}$ & $7.07^{\mathrm{a}}$ & $6.42^{\mathrm{a}}$ & $6.63^{\mathrm{a}}$ & $6.55^{\mathrm{a}}$ \\
\hline 0.71 & $0.64 / 0.62$ & $0.19 / 0.28$ & $0.43 / 0.36$ & $0.28 / 0.28$ & $6.78 / 6.96$ & $6.11 / 6.40$ & $6.47 / 6.51$ & $6.28 / 6.40$ \\
\hline 0.45 & $1.39^{\mathrm{a}}$ & $0.23^{\mathrm{b}} / 0.41^{\mathrm{b}}$ & $0.28^{\mathrm{b}} / 0.30^{\mathrm{b}}$ & $0.28^{\mathrm{b}} / 0.40^{\mathrm{b}}$ & $7.12^{\mathrm{a}}$ & $6.20^{\mathrm{b}} / 6.60^{\mathrm{b}}$ & $6.28^{\mathrm{b}} / 6.43^{\mathrm{b}}$ & $6.28^{\mathrm{b}} / 6.56^{\mathrm{b}}$ \\
\hline 0.14 & $0.72 / 0.58$ & $0.25 / 0.18$ & $0.36 / 0.34$ & $0.24 / 0.30$ & $6.83 / 6.93$ & $6.23 / 6.21$ & $6.39 / 6.49$ & $6.21 / 6.43$ \\
\hline
\end{tabular}

Notes. $\varphi$ Orbital phase (see Table 1)

${ }^{\text {a }}$ Data for the primary and secondary components not deblended.

${ }^{b}$ Data measured with low $\mathrm{S} / \mathrm{N}$.

Table 10

EW of the Different Chromospheric Activity Indicators of GZ Leo

\begin{tabular}{|c|c|c|c|c|c|c|c|c|c|c|c|}
\hline \multirow[t]{3}{*}{$\mathrm{O}$} & \multirow[t]{3}{*}{$\varphi$} & \multicolumn{10}{|c|}{ EW $(\AA)$ in the subtracted spectrum $(\mathrm{P} / \mathrm{S})$} \\
\hline & & \multicolumn{2}{|c|}{$\mathrm{Ca}$ II } & \multirow[b]{2}{*}{$\mathrm{H} \epsilon$} & \multirow[b]{2}{*}{$\mathrm{H} \delta$} & \multirow[b]{2}{*}{$\mathrm{H} \gamma$} & \multirow[b]{2}{*}{$\mathrm{H} \beta$} & \multirow[b]{2}{*}{$\mathrm{H} \alpha$} & \multicolumn{3}{|c|}{ Ca II IRT } \\
\hline & & $\mathrm{K}$ & $\mathrm{H}$ & & & & & & $\lambda 8498$ & $\lambda 8542$ & $\lambda 8662$ \\
\hline M & 0.12 & $\cdots$ & $\cdots$ & $\cdots$ & $\cdots$ & $\cdots$ & $\cdots$ & $0.29 / 0.30$ & $0.30 / 0.20$ & $0.22 / 0.21$ & $0.26 / 0.26$ \\
\hline M & 0.77 & $\ldots$ & $\cdots$ & $\ldots$ & $\ldots$ & $\ldots$ & $\ldots$ & $0.22 / 0.41$ & $0.23 / 0.33$ & $0.41 / 0.52$ & $0.37 / 0.40$ \\
\hline M & 0.46 & $\ldots$ & $\cdots$ & $\cdots$ & $\ldots$ & $\ldots$ & $\ldots$ & $0.29 / 0.42$ & c/0.49 & $0.90^{\mathrm{a}} / \mathrm{c}$ & $0.54^{\mathrm{a}} /^{\mathrm{c}}$ \\
\hline M & 0.11 & $\cdots$ & $\cdots$ & $\cdots$ & $\cdots$ & $\cdots$ & $\cdots$ & $0.29 / 0.28$ & $\cdots$ & $\cdots$ & $\cdots$ \\
\hline M & 0.76 & $\cdots$ & $\cdots$ & $\ldots$ & $\cdots$ & $\cdots$ & $\cdots$ & $0.26 / 0.34$ & $0.13 / 0.39$ & $0.33 / 0.29$ & \\
\hline M & 0.42 & $\cdots$ & $\ldots$ & $\ldots$ & $\cdots$ & $\cdots$ & $\cdots$ & $0.31 / 0.32$ & $0.21 / 0.25$ & $0.29 / 0.25$ & $0.27 / 0.34$ \\
\hline M & 0.08 & $\cdots$ & $\cdots$ & $\cdots$ & $\cdots$ & $\cdots$ & $\cdots$ & $0.34 / 0.40$ & $0.14 / 0.18$ & $0.35 / 0.30$ & $\mathrm{c} / \mathrm{c}$ \\
\hline M & 0.73 & $\ldots$ & $\cdots$ & $\cdots$ & $\cdots$ & $\cdots$ & $\cdots$ & $0.33 / 0.47$ & $0.13 / 0.28$ & $0.32 / 0.37$ & $0.24 / 0.23$ \\
\hline M & 0.39 & $\ldots$ & $\ldots$ & $\ldots$ & $\ldots$ & $\ldots$ & $\ldots$ & $0.44 / 0.38$ & $0.23 / 0.20$ & $0.37 / 0.38$ & $0.16 / 0.24$ \\
\hline M & 0.04 & $\ldots$ & $\ldots$ & $\ldots$ & $\ldots$ & $\ldots$ & $\ldots$ & $0.41 / 0.28$ & $0.18 / 0.32$ & $0.27 / 0.38$ & $0.22 / 0.41$ \\
\hline $\mathrm{F}$ & 0.68 & $1.06 / 1.02$ & $0.85 / 0.92^{\mathrm{e}}$ & $\mathrm{d} / 0.50$ & $0.09 / 0.13^{\mathrm{b}}$ & $\mathrm{c} / 0.08^{\mathrm{b}}$ & $0.12 / 0.08^{b}$ & $0.54 / 0.48$ & $0.33 / 0.35$ & $0.33 / 0.30$ & $0.37 / 0.35$ \\
\hline $\mathrm{F}$ & 0.36 & $0.77 / 0.84$ & $0.80^{\mathrm{e}} / 0.68$ & $0.17 /{ }^{\mathrm{d}}$ & $0.05 / 0.09^{b}$ & $0.08 / 0.10^{\mathrm{b}}$ & $0.09 / 0.15^{\mathrm{b}}$ & $0.32 / 0.52$ & $0.39 / 0.40$ & $0.33 / 0.37$ & $0.34 / 0.42$ \\
\hline $\mathrm{F}$ & 0.02 & $1.52^{\mathrm{a}}$ & $1.27^{\mathrm{a}}$ & $0.68^{\mathrm{a}}$ & $0.10^{\mathrm{a}, \mathrm{b}}$ & $0.10^{\mathrm{a}, \mathrm{b}}$ & $0.21^{\mathrm{a}, \mathrm{b}}$ & $0.74^{\mathrm{a}}$ & $0.60^{\mathrm{a}}$ & $0.50^{\mathrm{a}}$ & $0.78^{\mathrm{a}}$ \\
\hline $\mathrm{F}$ & 0.70 & $0.92 / 0.87$ & $0.92 / 0.59$ & $0.20 / 0.35$ & $0.07 / 0.08^{\mathrm{b}}$ & $0.12 / 0.09^{\mathrm{b}}$ & $0.18 / 0.16^{\mathrm{b}}$ & $0.49 / 0.43$ & $0.36 / 0.37$ & $0.33 / 0.32$ & $0.41 / 0.38$ \\
\hline $\mathrm{F}$ & 0.71 & $0.76 / 0.63$ & $0.70 / 0.68$ & $0.40 / 0.44$ & $0.08 / 0.08^{b}$ & $0.08 / 0.09^{b}$ & $0.13 / 0.13^{b}$ & $0.50 / 0.46$ & $0.36 / 0.37$ & $0.35 / 0.32$ & $0.40 / 0.37$ \\
\hline
\end{tabular}

Notes. O Observing run: M: McDonald98, F: FOCES02. $\varphi$ Orbital phase (see Table 1).

${ }^{a}$ Data for the primary and secondary components not deblended.

${ }^{b}$ Mean value of measured (from two apertures in our spectra) or value from higher $\mathrm{S} / \mathrm{N}$ aperture.

${ }^{c}$ Data not measured due to very low $\mathrm{S} / \mathrm{N}$.

${ }^{\mathrm{d}}$ Data not measured due to blending.

${ }^{\text {e }} \mathrm{Ca}$ II $\mathrm{H}$ line of one component blended with $\mathrm{H} \epsilon$ of the other component.

The absorption lines were clearly filled-in with emission in the observed spectra. After applying the spectral-subtraction, we could extract the excess emission from both components (see representative spectra in Figure 6). When the $\mathrm{S} / \mathrm{N}$ was high enough we deblended the emission coming from both components by applying a two-Gaussian fit to the subtracted spectra (see Table 10). These three lines show similar variations with orbital phase to the $\mathrm{H} \alpha$ line in both components, their mean values are $\mathrm{EW}(\mathrm{H} \beta)=0.13 / 0.13 \AA, \mathrm{EW}(\mathrm{H} \gamma)=0.09 / 0.09 \AA$ and $\mathrm{EW}(\mathrm{H} \delta)=0.07 / 0.09 \AA$.

We also measured the ratio of excess emission EW in the $\mathrm{H} \alpha$ and $\mathrm{H} \beta$ lines, $\mathrm{EW}(\mathrm{H} \alpha) / E W(\mathrm{H} \beta)$, and the ratio of excess emission $E_{\mathrm{H} \alpha} / E_{\mathrm{H} \beta}$ with the correction:

$$
\frac{E_{\mathrm{H} \alpha}}{E_{\mathrm{H} \beta}}=\frac{\mathrm{EW}(\mathrm{H} \alpha)}{\mathrm{EW}(\mathrm{H} \beta)} * 0.2444 * 2.512^{(B-R)}
$$

from Hall \& Ramsey (1992). It takes into account the absolute flux density in these lines and the color difference in the components ( $B-R=1.45$ for a K0V star). We obtained a mean values of $E_{\mathrm{H} \alpha} / E_{\mathrm{H} \beta} \approx 3.4$ for the primary and $\approx 3.6$ for secondary. These values indicate, according to Buzasi (1989) and Hall \& Ramsey (1992) the presence of prominence-like material at the stellar surface in both components of the system.

\section{3. $\mathrm{Ca}$ II $H \& \mathrm{~K}$ and $H \epsilon$}

The $\mathrm{Ca}$ II $\mathrm{H} \& \mathrm{~K}$ line region was included only in the spectra of the FOCES run for GZ Leo. In these spectra we observed strong emission in the $\mathrm{Ca}$ II $\mathrm{H} \& \mathrm{~K}$ lines and a clear emission in the $\mathrm{H} \epsilon$ line arising from both components (see Figure 4).

In our spectra, the $\mathrm{Ca}$ II $\mathrm{H} \& \mathrm{~K}$ lines are located at the end of the echellogram, where the efficiency of the spectrograph and the CCD decrease very rapidly, and therefore the $\mathrm{S} / \mathrm{N}$ 

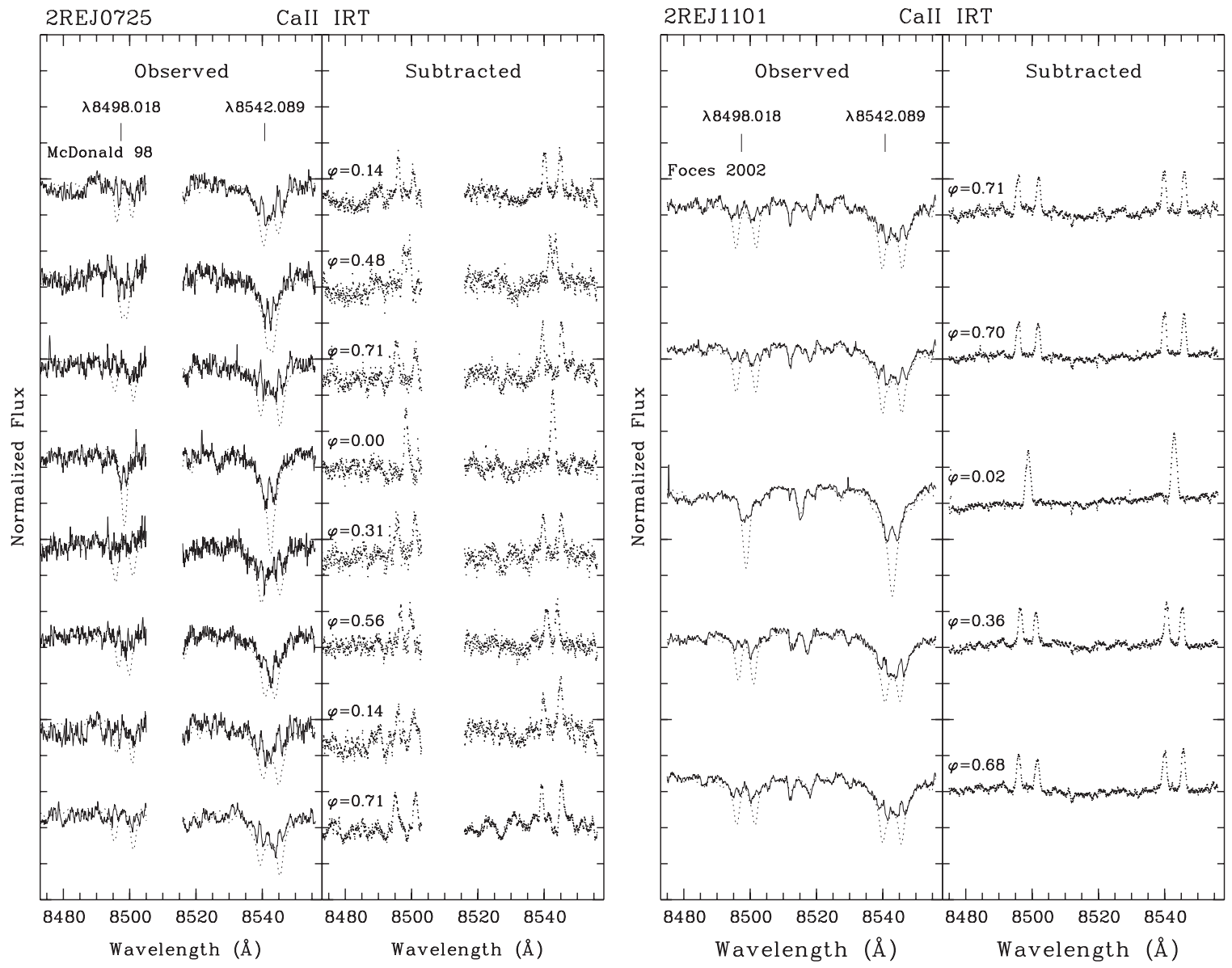

Figure 5. Same as previous figure but in the region of the Ca II IRT $(8498,8542 \AA)$ lines. V789 Mon (2RE J0725) system on the left in McDonald run and GZ Leo (2RE J1101) on the right in FOCES run. An excess emission from the primary (P) and secondary (S) components is detected and marked.

Table 11

Absolute Surface Flux of the Different Chromospheric Indicators of GZ Leo

\begin{tabular}{|c|c|c|c|c|c|c|c|c|c|c|c|}
\hline \multirow[t]{3}{*}{$\overline{\mathrm{O}}$} & \multirow[t]{3}{*}{$\bar{\varphi}$} & \multicolumn{10}{|c|}{$\log F_{\mathrm{S}}(\mathrm{P} / \mathrm{S})$} \\
\hline & & \multicolumn{2}{|c|}{ Ca II } & \multirow[b]{2}{*}{$\mathrm{H} \epsilon$} & \multirow[b]{2}{*}{$\mathrm{H} \delta$} & \multirow[b]{2}{*}{$\mathrm{H} \gamma$} & \multirow[b]{2}{*}{$\mathrm{H} \beta$} & \multirow[b]{2}{*}{$\mathrm{H} \alpha$} & \multicolumn{3}{|c|}{ Ca II IRT } \\
\hline & & $\mathrm{K}$ & $\mathrm{H}$ & & & & & & $\lambda 8498$ & $\lambda 8542$ & $\lambda 8662$ \\
\hline M & 0.77 & $\cdots$ & $\ldots$ & $\ldots$ & $\ldots$ & $\ldots$ & $\ldots$ & $6.40 / 6.67$ & $6.25 / 6.41$ & $6.50 / 6.61$ & $6.46 / 6.49$ \\
\hline M & 0.46 & $\ldots$ & $\ldots$ & $\ldots$ & $\ldots$ & $\ldots$ & $\ldots$ & $6.52 / 6.68$ & c/6.58 & $6.84^{\mathrm{a}} /{ }^{\mathrm{c}}$ & $6.62^{\mathrm{a}} /^{\mathrm{c}}$ \\
\hline M & 0.11 & $\ldots$ & $\ldots$ & $\ldots$ & $\ldots$ & $\ldots$ & $\ldots$ & $6.52 / 6.51$ & $\ldots$ & $\ldots$ & $\ldots$ \\
\hline M & 0.08 & $\cdots$ & $\ldots$ & $\ldots$ & $\ldots$ & $\ldots$ & $\ldots$ & $6.59 / 6.66$ & $6.04 / 6.15$ & $6.43 / 6.37$ & $\mathrm{c} / \mathrm{c}$ \\
\hline M & 0.73 & $\ldots$ & $\ldots$ & $\ldots$ & $\ldots$ & $\ldots$ & $\ldots$ & $6.58 / 6.73$ & $6.00 / 6.34$ & $6.40 / 6.46$ & $6.27 / 6.25$ \\
\hline M & 0.39 & $\ldots$ & $\ldots$ & $\ldots$ & $\ldots$ & $\ldots$ & $\ldots$ & $6.70 / 6.64$ & $6.25 / 6.19$ & $6.46 / 6.47$ & $6.09 / 6.27$ \\
\hline $\mathrm{M}$ & 0.04 & $\ldots$ & $\ldots$ & $\ldots$ & $\ldots$ & $\ldots$ & $\ldots$ & $6.67 / 6.51$ & $6.15 / 6.40$ & $6.32 / 6.47$ & $6.23 / 6.50$ \\
\hline $\mathrm{F}$ & 0.68 & $7.11 / 7.09$ & $7.01 / 7.05^{\mathrm{e}}$ & $\mathrm{d} / 6.78$ & $6.04 / 6.20^{\mathrm{b}}$ & c $/ 5.98^{2}$ & $6.15 / 5.98^{b}$ & $6.79 / 6.74$ & $6.41 / 6.43$ & $6.41 / 6.37$ & $6.46 / 6.43$ \\
\hline $\mathrm{F}$ & 0.36 & $6.97 / 7.01$ & $6.99^{\mathrm{e}} / 6.92$ & $6.32 /^{\mathrm{d}}$ & $5.78 / 6.04^{b}$ & $5.98 / 6.08^{b}$ & $6.03 / 6.25^{b}$ & $6.57 / 6.78$ & $6.48 / 6.49$ & $6.41 / 6.45$ & $6.42 / 6.51$ \\
\hline
\end{tabular}

Note. Notes as in Table 10.

ratio obtained is very low, and the normalization of the spectra are very difficult. In spite of this we could apply the spectral subtraction in this region, see Figure 4. As we can see in this figure, the $\mathrm{H} \epsilon$ line arising from one of the component overlaps at some orbital phases with the $\mathrm{Ca}$ II $\mathrm{H}$ line from the other component, so their EW were measured with a Gaussian fit when it was possible (see comments for each spectrum in the footnotes of Table 10).

The mean measured EWs in these spectra are $\mathrm{EW}=$ $1.13 / 0.84 \AA$ for each component in the $\mathrm{Ca}$ II $\mathrm{K}$ line and $\mathrm{EW}=$ $0.82 / 0.65 \AA$ for each component in the Ca II $\mathrm{H}$ line. An increase of the $\mathrm{Ca}$ II $\mathrm{H} \& \mathrm{~K}$ emissions is observed with the orbital phase. 


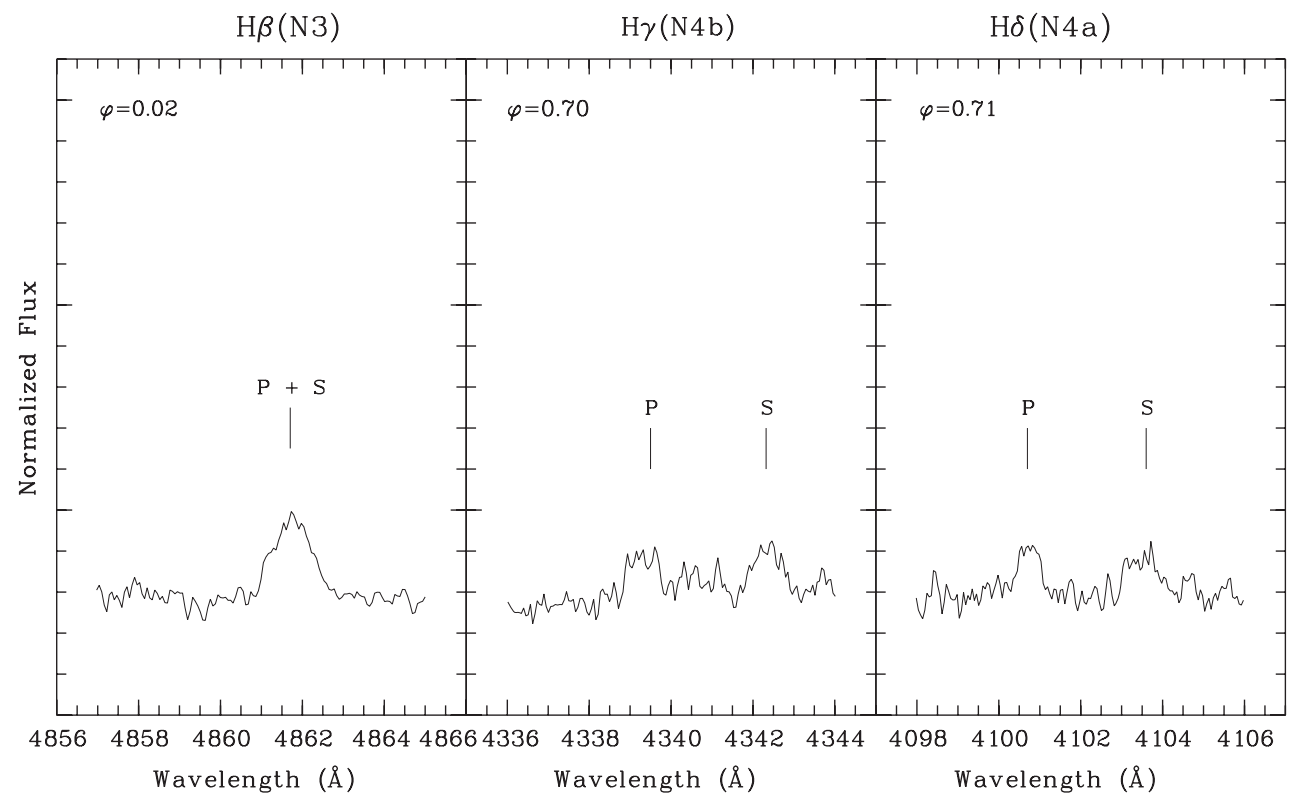

Figure 6. Subtracted spectra in the region of the $\mathrm{H} \beta, \mathrm{H} \gamma$, and $\mathrm{H} \delta$ lines for GZ Leo in FOCES observing run. Clear excess emission from the primary (P) and secondary (S) components is detected.

The presence of an excess emission from $\mathrm{H} \epsilon$ line (EW= $0.30 / 0.43 \AA$ ) indicates that it is a very active system.

\subsection{Ca II IRT Lines ( $\lambda 8498, \lambda 8542$, and $\lambda 8662)$}

The three lines of the Ca II infrared triplet (IRT) were included in all our echelle spectra for both systems. In all of them we could observe a clear emission above the continuum in the core of the Ca II IRT absorption lines (see Figure 5) from both components.

In V789 Mon, (Figure 5 left side), we measured an average $\mathrm{EW}$ of $\approx 0.4 \AA$ for the three lines, and we found small variations with the orbital phase that were anticorrelated with the Balmer lines EW variations in both primary and secondary components.

For GZ Leo binary, (Figure 5 right side), we found that in FOCES run there were no significant variations with the orbital phase, but in McDonald's one an evident orbital variation were observed which shows an anticorrelation with the $\operatorname{EW}(\mathrm{H} \alpha)$ variation. We measured mean $\mathrm{EWs}$ of $\approx 0.25 / 0.31 \AA$ for each component in McDonald run and $\approx 0.36 \AA$ for both components in FOCES run.

In addition, we calculated the ratio of excess emission EW, $E_{8542} / E_{8498}$, which is also an indicator of the type of chromospheric structure that produces the observed emission. In solar plages, values of $E_{8542} / E_{8498} \approx 1.5-3$ are measured, while in solar prominences the values are $\approx 9$, the limit of an optically thin emitting plasma (Chester 1991).

In V789 Mon, small values of the $E_{8542} / E_{8498}$ ratio were found, $\approx 1.5$ for the primary component and 1.4 for the secondary (see Table 9). This indicates that the Ca II IRT emission of this star arises from plage-like regions.

In GZ Leo, we found a $E_{8542} / E_{8498}$ value of $\approx 2.4$ and $\approx 1.4$ for primary and secondary in McDonald run, but $\approx 0.9$ for primary and secondary in FOCES run. These values indicate that $\mathrm{Ca}$ II IRT emission come from plage-like regions, in contrast to the Balmer lines which seem to come from prominences.

This markedly different behavior of the Ca II IRT and the $\mathrm{H} \alpha$ emission has also been found in other chromospherically active binaries (see Arévalo \& Lázaro 1999; Montes et al. 2000; Gálvez et al. 2003; Gálvez 2005 and references therein). The different behavior from one epoch to another is consistent with the different activity level measured in each observing run. The lack of variation with orbital phase of the GZ Leo emission lines in FOCES run could be due to that the high level of activity is reflecting a large number of active regions maybe distributed along all surface. Such, could produce an even spotted-plaged surface that would avoid to see the modulation clearly.

\section{SUMMARY}

In this paper, we present a detailed spectroscopic analysis of two X-ray/EUV selected chromospherically active binary systems V789 Mon (2RE J0725-002) and GZ Leo (2RE $\mathrm{J} 1101+223)$. We analyzed high-resolution echelle spectra that include the optical chromospheric activity indicators from the $\mathrm{Ca}$ II $\mathrm{H} \& \mathrm{~K}$ to $\mathrm{Ca}$ II IRT lines, as well as the $\mathrm{Li}$ I $\lambda 6707.8$ line and other photospheric lines of interest.

Using a large number of radial velocities we improved the orbital parameters of these systems. We obtained that both systems have nearly circular orbits and have an orbital period very close to photometric period, indicating that they have a synchronous rotation.

The spectral classification derived using the comparison with spectra of reference stars, two K5V components for V789 Mon and two K0V components for GZ Leo is in good agreement with the classification obtained from physical parameters $(R \sin i)$. As both components are main sequence stars, we can classify these chromospherically active binaries as BY Dra systems (Fekel et al. 1986).

By using the information provided by the width of the CCF, we determined a projected rotational velocity, $v \sin i$, of 28.28 and $25.09 \mathrm{~km} \mathrm{~s}^{-1}$ for the primary and secondary components of V789 Mon, and 26.23 and $26.98 \mathrm{~km} \mathrm{~s}^{-1}$ for the components of GZ Leo.

The presence of the $\mathrm{Li}$ I line in both systems is in agreement with the kinematics results, i.e., they belong to the young disk population. Also, with a EW(Li I $) \approx 40 \mathrm{~m} \AA$ for each component, GZ Leo could be a member of the Hyades supercluster.

We analyzed, using the spectral-subtraction technique, all the optical chromospheric activity indicators. In V789 Mon, 
both components show high levels of chromospheric activity. The emission in the $\mathrm{H} \alpha$ line is above the continuum and it is very similar in the two components, showing no variations with phase. GZ Leo shows a high level of chromospheric activity too. After applying spectral subtraction the chromospheric excess emission from both components are certainly detected in all the activity indicators during the observing runs. The $\mathrm{Ca}$ II $\mathrm{H} \& \mathrm{~K}$ lines are in emission and $\mathrm{H} \epsilon$ is also detected in emission for both systems.

The level of chromospheric emission change, i.e., we have observed a $35 \%$ increment in the $\mathrm{EW}$ of $\mathrm{H} \alpha$ emission line and a nearly $30 \%$ increment in the EW of Ca II IRT emission lines from one epoch to another.

Furthermore, the variation of $\mathrm{H} \alpha$ and $\mathrm{Ca}$ II IRT emission with phase is anticorrelated and the ratios $E_{\mathrm{H} \alpha} / E_{\mathrm{H} \beta}$ and $E_{8542} / E_{8498}$ indicate that the emission of the Balmer lines would arise from prominence-like material, and that the emission of Ca II IRT lines arise from plage-like regions.

We thank Dr. L.W. Ramsey (Pennsylvania State University) for collaborating in the McDonald observing run $(2.1 \mathrm{~m}$ telescope, Texas, USA) and the staff of McDonald Observatory for their allocation of observing time and their assistance with our observations. We thank Dr. Nadya Gorlova and Dr. Joern Rossa for their great help on the English redaction and corrections. This work was supported by the Spanish "Programa Nacional de Astronomía y Astrofísica" under grants AYA200502750 and AYA2008-00695, and the "Comunidad de Madrid" under PRICIT project S-0505/ESP-0237 (ASTROCAM).

\section{REFERENCES}

Arévalo, M. J., \& Lázaro, C. 1999, AJ, 118, 1015

Barden, S. C. 1985, ApJ, 295, 162

Beavers, W. I., Eitter, J. J., Ketelsen, D. A., \& Oesper, D. A. 1979, PASP, 91, 698

Buzasi, D. L. 1989, PhD thesis, Pennsylvania State Univ.

Chester, M. M. 1991, PhD thesis, Pennsylvania State Univ.

Cutispoto, G., Pastori, L., Tagliaferri, G., Messina, S., \& Pallavicini, R. 1999, A\&AS, 138, 87
Eggen, O. J. 1984, ApJS, 55, 597

Eggen, O. J. 1989, PASP, 101, 366

ESA 1997, The Hipparcos and Tycho Catalogues, ESA SP-1200

Favata, F., Barbera, M., Micela, G., \& Sciortino, S. 1993, A\&A, 277, 428

Fekel, F. C., Bopp, B. W., Africano, J. L., Goodrich, B. D., Palmer, L. H., Quigley, R., \& Simon, T. 1986, AJ, 92, 1150

Fekel, F. C., \& Henry, G. W. 2000, ApJ, 120, 3265

Gálvez, M. C. 2005, PhD thesis, Universidad Complutense de Madrid

Gálvez, M. C., Montes, D., Fernández-Figueroa, M. J., López-Santiago, J., De Castro, E. \& Cornide, M. 2002, A\&A, 389, 524 (Paper IV)

Gálvez, M. C., Montes, D., Ramsey, L. W., Fernández-Figueroa, M. J., De Castro, E., \& Cornide, M. 2003, in Proc. 5th Scientific Meeting of the Spanish Astronomical Society (SEA), Highlights of Spanish Astrophysics III, ed. J. Gallego, J. Zamorano, \& N. Cardiel (Dordrecht: Kluwer) 474

Gálvez, M. C., Montes, D., Fernández-Figueroa, M. J., De Castro, E., \& Cornide, M. 2007, A\&A, 472, 587 (Paper V)

Hall, J. C. 1996, PASP, 108, 313

Hall, J. C., \& Ramsey, L. W. 1992, AJ, 104, 1942

Høg, E., et al. 2000, A\&A, 355, L27

Jeffries, R. D., Bertram, D., \& Spurgeon, B. R. 1995, MNRAS, 276, 397

López-Santiago, J., Montes, D., Fernández-Figueroa, M. J., \& Ramsey, L. W. 2003, A\&A, 411, 489

Montes, D., De Castro, E., Fernádez-Figueroa, M. J., \& Cornide, M. 1995, A\&AS, 114, 287

Montes, D., Fernández-Figueroa, M. J., De Castro, E., Cornide, M., Latorre, A., \& Sanz-Forcada, J. 2000, A\&AS, 146, 103 (Paper III)

Montes, D., Fernández-Figueroa, M. J., De Castro, E., \& Sanz-Forcada, J. 1997, A\&AS, 125, 263 (Paper I)

Montes, D., López-Santiago, J., Fernández-Figueroa, M. J., \& Gálvez, M. C. 2001a, A\&A, 379, 976

Montes, D., López-Santiago, J., Gálvez, M. C., Fernández-Figueroa, M. J., De Castro, E., \& Cornide, M. 2001b, MNRAS, 328, 45

Montes, D., Sanz-Forcada, J., Fernández-Figueroa, M. J., De Castro, E., \& Poncet, A. 1998, A\&A, 330, 155 (Paper II)

Pandey, J. C., Singh, K. P., Drake, S. A., \& Sagar, R. 2005, AJ, 130, 1231

Pandey, J. C., Singh, K. P., Sagar, R., \& Drake, S. A. 2002, J. Astrophys. Astron., 23, 9

Popper, D. M. 1996, ApJSS, 106, 133

Press, W. H., Flannery, B. P., \& Teukolsky, S. A. 1986, Numerical Recipes. The Art of Scientific Computing (Cambridge: Cambridge Univ. Press)

Robb, R. M., \& Gladders, M. D. 1996, IAU, 4412

Schmidt-Kaler, T. 1982, in Landolt-Börnstein,Vol. 2b, ed. K. Schaifers \& H. H. Voig (Heidelberg: Springer)

Strassmeier, K. G., Washuettl, A., Granzer, Th., Scheck, M., \& Weber, M. 2000, A\&AS, 142, 275

Tonry, J., \& Davis, M. 1979, AJ, 84, 1511 